\title{
L'art au service de la conservation de l'un des lémuriens les plus menacés
}

\section{Art for the conservation of one of the most endangered lemurs}

\author{
Delphine Roullet ${ }^{1}$ \\ ${ }^{1}$ Fondatrice \& Présidente d'Helpsimus, helpsimus@yahoo.fr
}

RÉSUMÉ. HELPSIMUS s'est donnée pour mission de sauvegarder le Grand Hapalémur (Prolemur simus) à Madagascar à travers le programme Bamboo Lemur qui associe un suivi scientifique de l'espèce, la protection de son habitat, l'aide au développement des villages riverains des groupes de grands hapalémurs et le financement de l'éducation des enfants. En 2013, nous avons créé la " Fête du Simus " qui est devenu un évènement annuel incontournable dans la région où Helpsimus intervient. Au cours de cette journée de célébration, les enfants des 5 écoles primaires publiques localisées sur le site du programme Bamboo Lemur, mais aussi des groupes locaux issus des villages avec lesquels nous collaborons, sont invités à présenter les créations artistiques qu'ils ont préparé pour l'occasion : danses, chants et pièces de théâtre sur les grands hapalémurs et leur protection. Nous travaillons également avec un peintre malgache qui a réalisé plusieurs fresques sur les grands hapalémurs dans ces mêmes écoles. De plus, notre volet éducation n'est pas limité à Madagascar. Depuis 2015, il s'étend à plusieurs écoles situées dans de petites villes françaises. Un échange de lettres a d'abords commencé entre les écoles qui a été suivi par un soutien matériel aux écoles malgaches, lorsque les enfants français ont découvert les difficultés auxquelles étaient confrontés leurs nouveaux camarades dans leur vie quotidienne. Les enfants français ont alors décidé d'organiser des collectes de divers matériels et de fonds qui ont notamment permis de financer l'ouverture d'une cantine scolaire. Ils ont aussi créé une pièce originale sur les liens qu'ils ont tissés avec les enfants malgaches.

En France, nous avons organisé quelques expositions avec plusieurs artistes : photographes et peintres.

Enfin, nous développons un projet artisanal dans le cadre de notre programme d'écotourisme. Un bracelet original a ainsi été créé par une créatrice française à partir de graines locales.

Le programme Bamboo Lemur a été à l'origine de diverses créations artistiques impliquant des personnes différentes autour du même objectif celui de la protection d'un des lémuriens les plus menacés à Madagascar.

ABSTRACT. The Bamboo lemur program led by Helpsimus is based in southeast Madagascar, near Ranomafana National Park, within an unprotected and intensively cultivated area that is home to the largest wild population of greater bamboo lemur, Prolemur simus. Key elements are combined to guarantee its long-term success: scientific monitoring of the species, habitat protection, transfer of management responsibility to local communities, development aid for local communities, support to children's education. Our education component also includes environmental awareness. A "Simus Day" was created in 2013 and has since become the biggest annual festive event in the area where Helpsimus operates. During this day of celebration, the children of the 5 schools but also local bands from the villages with which we collaborate, are invited to perform dance, music and theatre play on greater bamboo lemurs and their protection.

We also work with a Malagasy painter who has created several frescoes on the greater bamboo lemurs in the primary schools supported by Helpsimus. Moreover, our education component is not restricted to Madagascar. Since 2015, it extends to several French schools located in small French cities. It begun with exchange of letters between schools which was soon followed by a support to Madagascan schools when the French children discovered the problems faced by their new fellows in their daily life. The French children decided to collect various materials and organised fundraising events that contributed, in particular, to finance the opening of a school canteen. Then, they have created an original play about the connections they have built with the Malagasy children.

In France, we organised a couple of exhibitions with several artists: photographers and painters.

Finally, we are developing a craft project as a part of our ecotourism program including the production of an original bracelet from local seeds, imagined by a French designer.

The program Bamboo Lemur led to the emergence of various artistic performances and projects involving different people for the same goal the protection of a very rare lemur.

MOTS-CLÉS. art, éducation, lémurien, école, conservation, sensibilisation, partage.

KEYWORDS. art, education, lemur, school, conservation, awareness, sharing. 
Le Grand Hapalémur (Prolemur simus) est classé en danger critique d'extinction sur la Liste Rouge de l'UICN. Il est l'un des lémuriens les plus menacés à Madagascar. Autrefois largement répandu dans toute l'île, on ne le trouve plus aujourd'hui que dans les forêts humides de l'Est de Madagascar, où un peu plus d'un millier d'individus ont été recensés (Helpsimus, 2015). Le grand hapalémur se nourrit presque exclusivement de bambou dont il consomme toutes les parties.

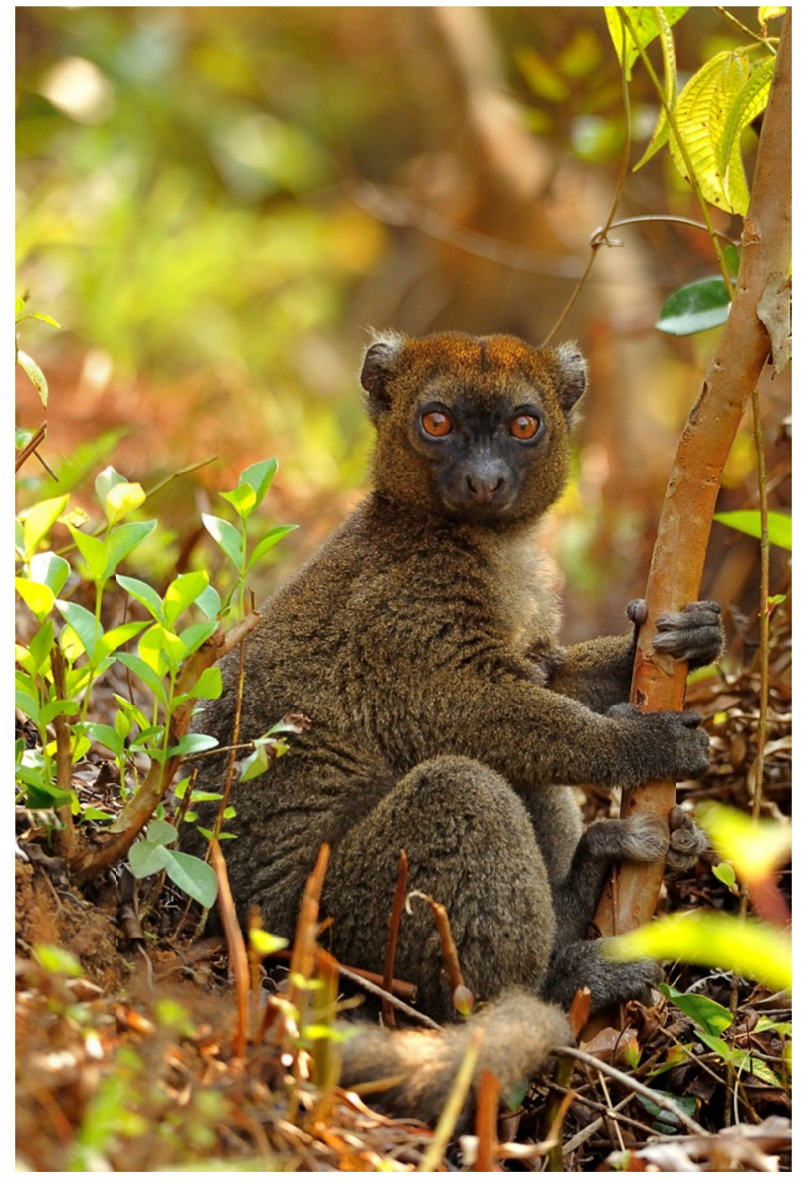

Photo 1. Grand Hapalémur $\odot$ F-G Grandin

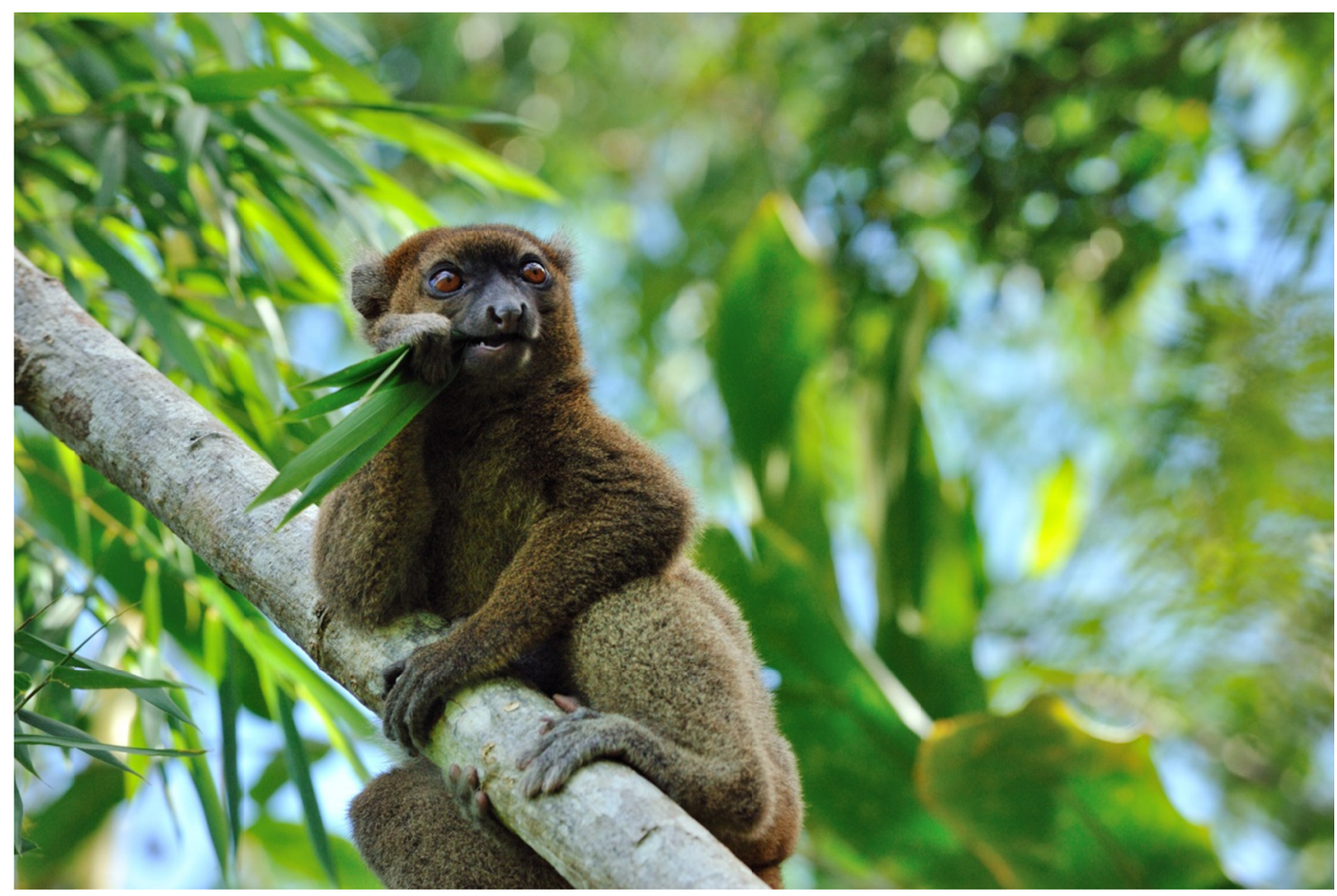

Photo 2. Grand hapalémur s'alimentant de feuilles de bambou $®$ F-G Grandin 
En 2008, alors qu'on pense l'espèce au bord de l'extinction avec moins d'une centaine d'individus subsistant à l'état sauvage (Wright et col., 2008), une nouvelle population est découverte au sud-est de Madagascar, à environ $4 \mathrm{~km}$ du Parc National de Ranomafana, à proximité de plusieurs villages de la commune rurale de Tsaratanana.

L'Association Française pour la Sauvegarde du Grand Hapalémur (ou "Helpsimus") est créée l'année suivante pour tenter de sauvegarder cette population nouvellement identifiée. Helpsimus met en place le programme Bamboo Lemur qui associe un suivi scientifique des animaux, la protection de leur habitat mais aussi l'aide au développement des villages riverains des groupes de grands hapalémurs et le financement de l'éducation des enfants.

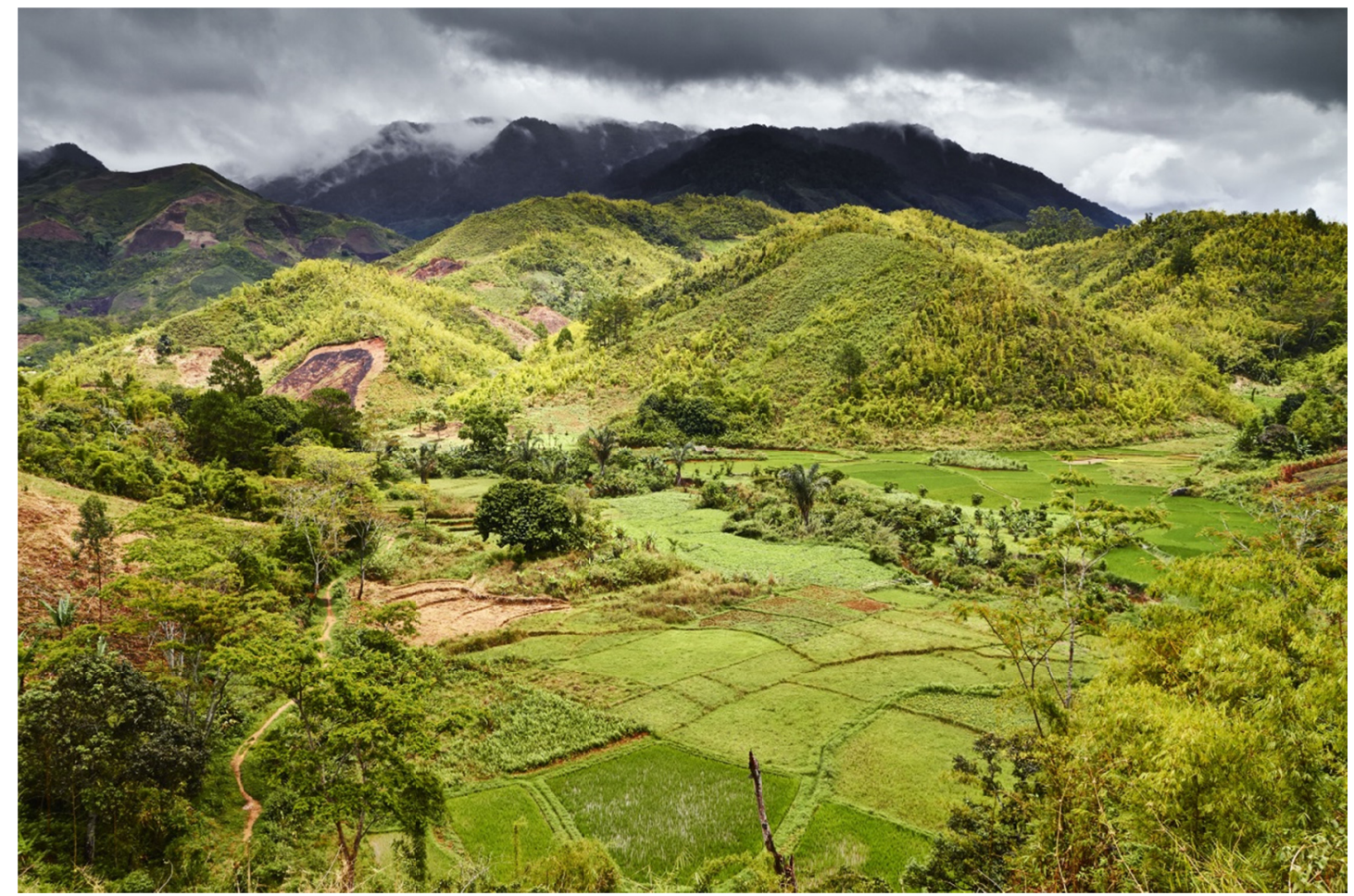

Photo 3. Site du programme Bamboo lemur Vohitrarivo @ S. Meys

Helpsimus protège aujourd'hui la plus importante population sauvage de grands hapalémurs (près de 400 individus) ainsi que son habitat constitué de bambous et de plusieurs fragments de forêts extrêmement dégradés par la pratique du " tavy » (culture sur brûlis) largement utilisée par les communautés locales qui dépendent entièrement des terres qu'elles cultivent. 


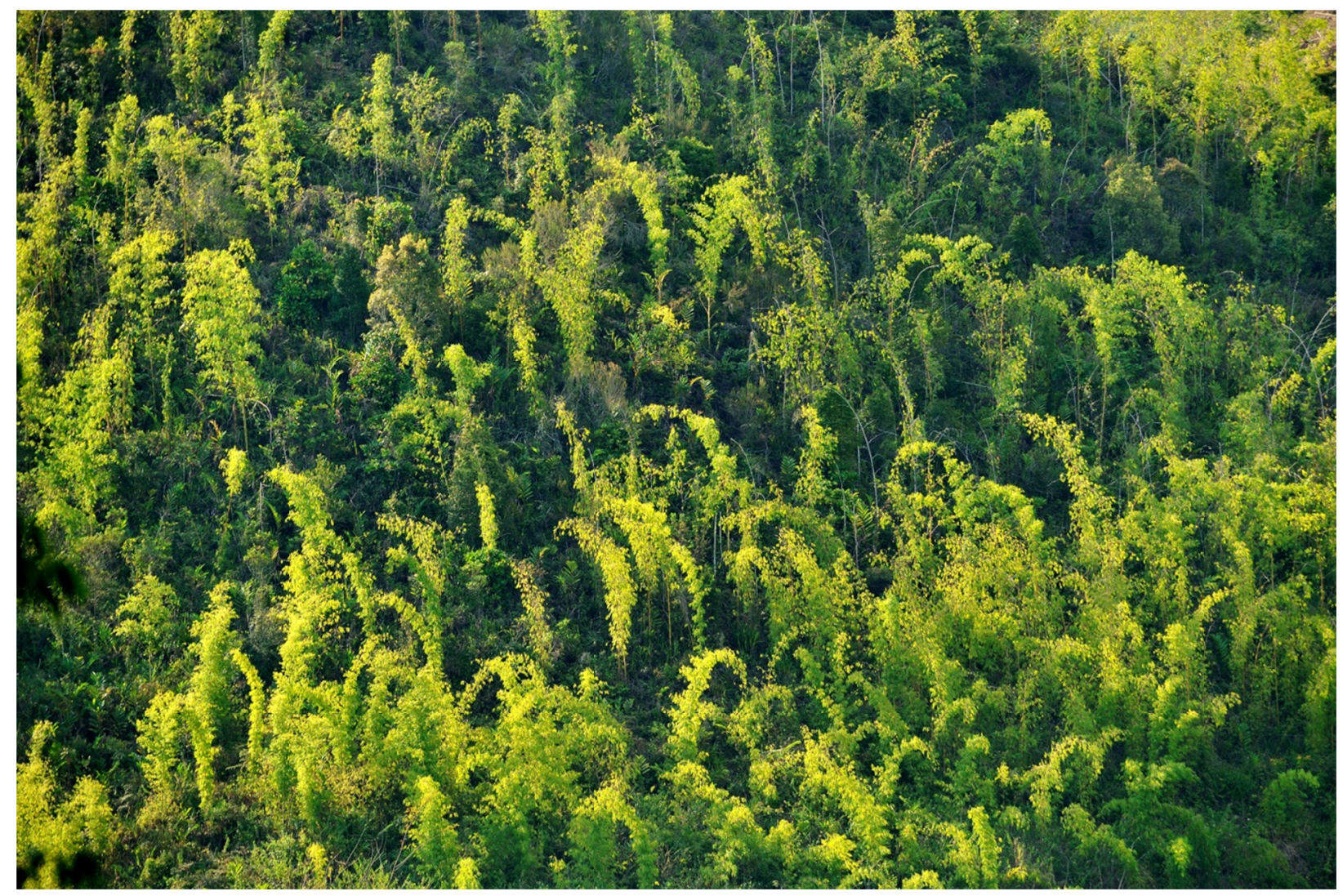

Photo 4. Forêt de bambou, site du programme Bamboo lemur (c) F-G Grandin

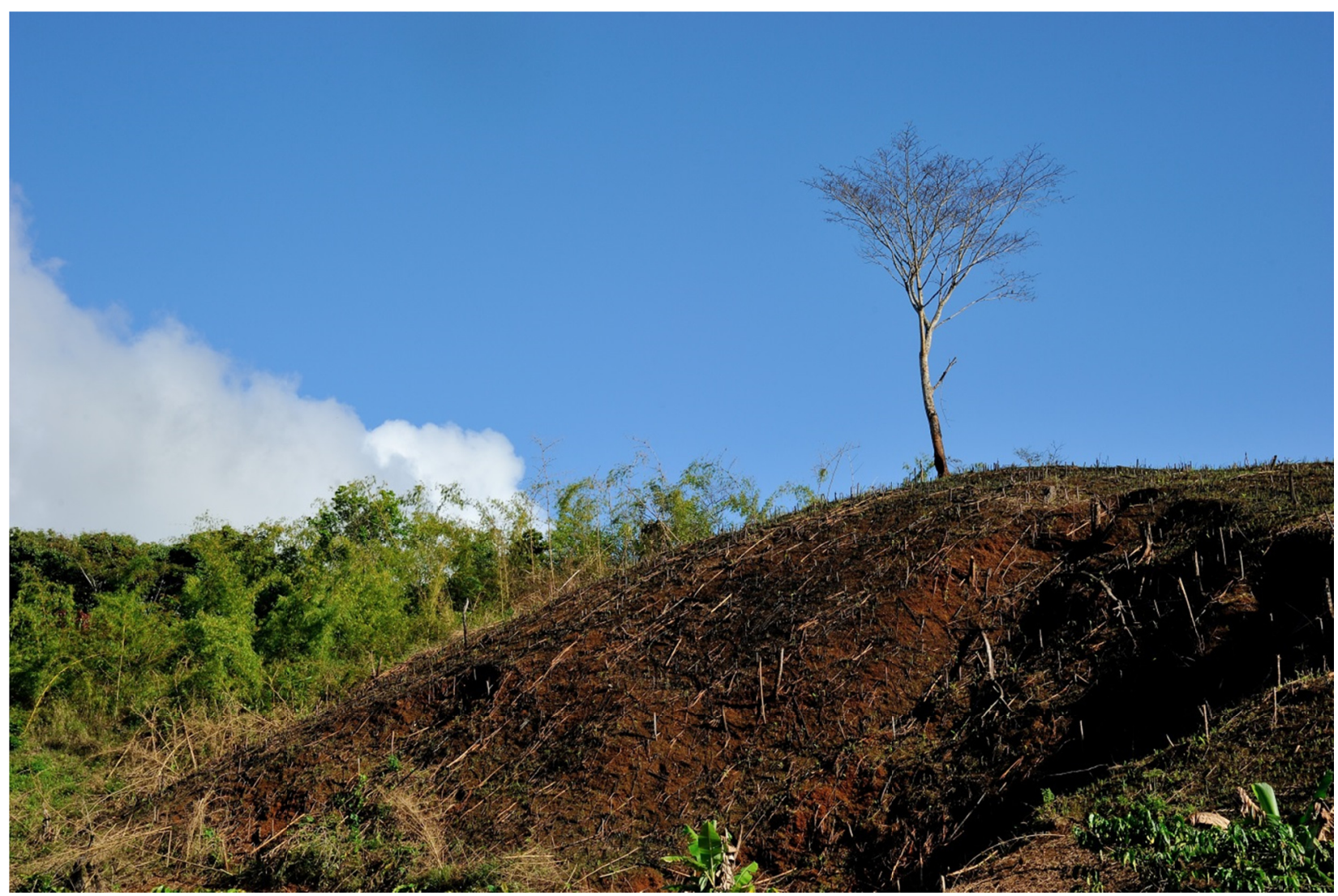

Photo 5. Tavy, Site du programme Bamboo lemur ${ }^{\circledR}$ F-G Grandin Helpsimus

L’objectif du programme Bamboo Lemur est de trouver un équilibre entre les besoins des hommes et ceux des lémuriens afin de faire cohabiter harmonieusement les communautés locales et la faune sauvage, en mettant en place des mesures de protection adaptées. 
Ces mesures, destinées à assurer la survie à long terme des grands hapalémurs, s'articulent autour de 3 volets :

- un volet environnemental : il cherche à améliorer la connaissance de l'éco-éthologie de l'espèce et à protéger son habitat. Une équipe de 20 guides locaux est notamment en charge du suivi des grands hapalémurs avec pour missions de localiser les animaux et de déterminer leur territoire (enregistrement des coordonnées GPS), de recenser les individus (mises à jour régulières des inventaires), les menaces (présence de chiens ou de pièges) ainsi que les incursions des lémuriens dans des cultures. Cette partie comprend également le reboisement des zones forestières les plus dégradées et la mise place de systèmes de protection des cultures afin de limiter les conflits hommes-lémuriens. De plus, la création de VOI (associations villageoises à qui l'Administration forestière malgache transfère la gestion des ressources naturelles) donne un premier niveau de protection à l'habitat des lémuriens à travers l'établissement d'un plan d'aménagement du site identifiant des zones de conservation et des zones d'exploitation ;

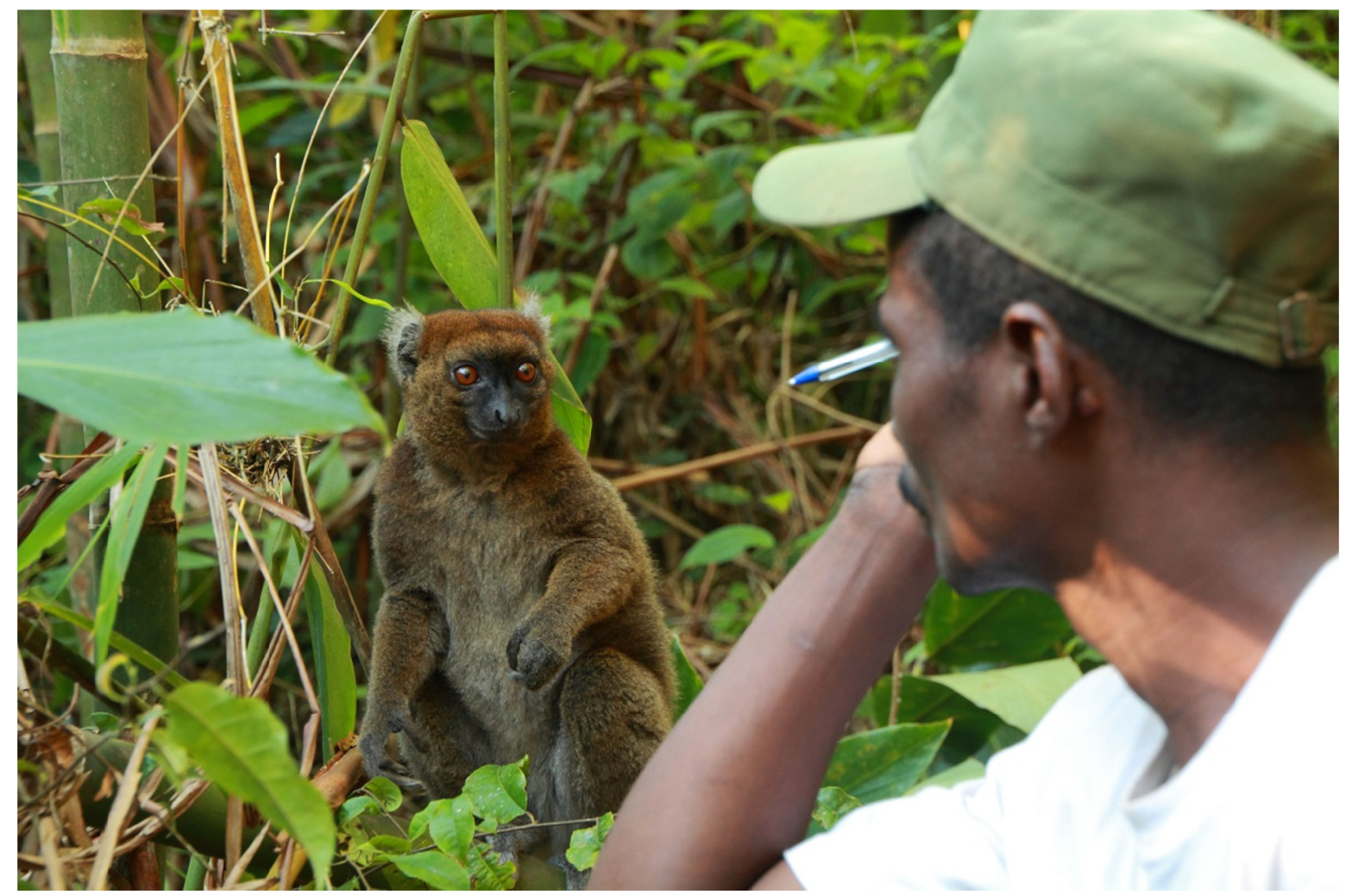

Photo 6. Suivi des lémuriens par des guides locaux () J. Catinaud 


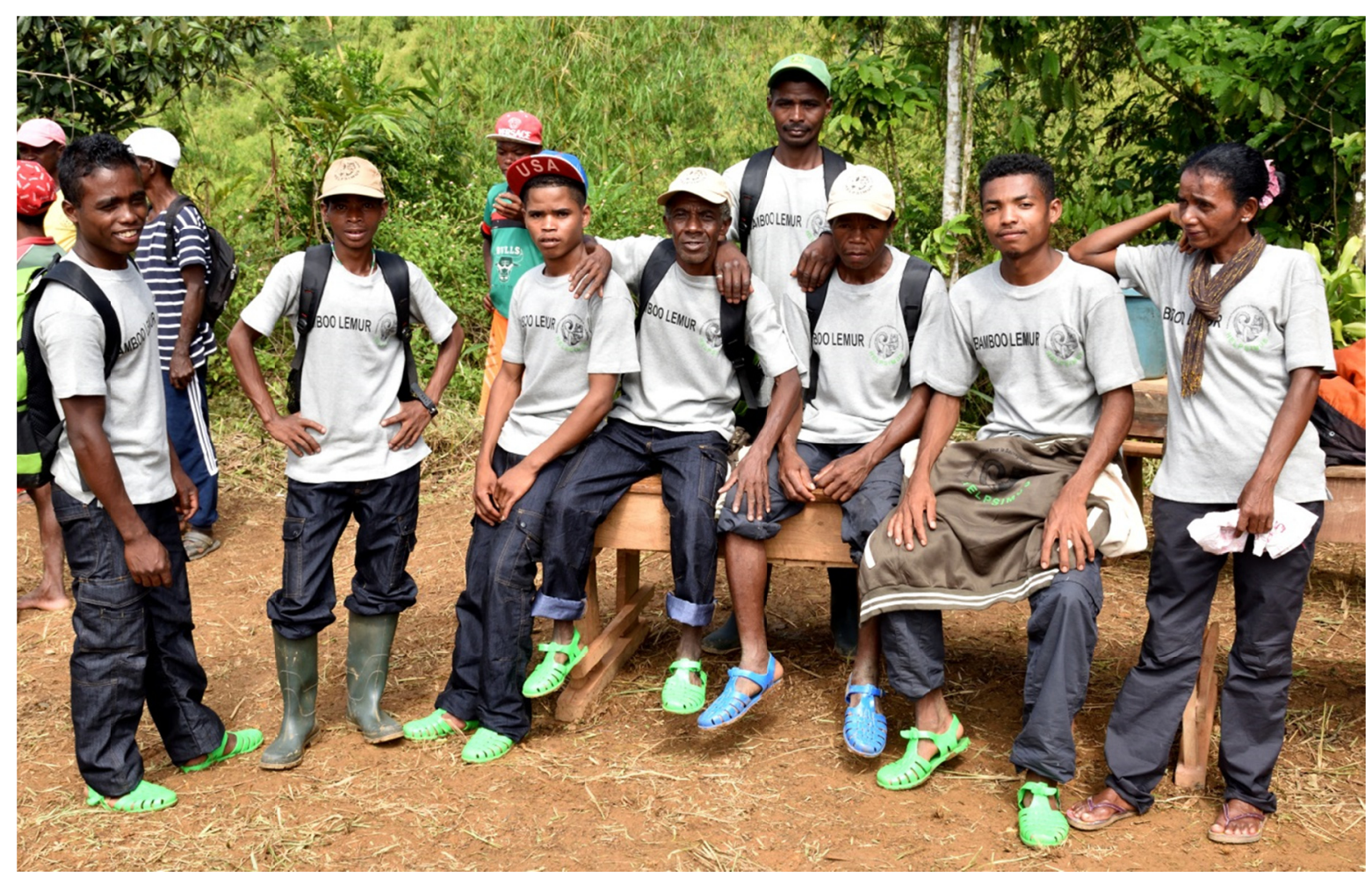

Photo 7. Une partie de l'équipe des guides 도. Roullet

- un volet socio-économique : il cherche à augmenter le niveau de vie des populations locales en soutenant des actions qui sécurisent les produits de leurs récoltes et améliorent les rendements, en développant de nouvelles Activités Génératrices de Revenus, en construisant des infrastructures et des moyens de communication et en encourageant de meilleures pratiques d'hygiène pour favoriser une meilleure santé des communautés. Helpsimus finance ainsi des formations diverses : Système de Riziculture améliorée, valorisation du longoza, artisanat, fonctionnement d'une association etc. Enfin nous avons lancé un projet d'écotourisme, qui, avec l'ensemble des autres activités contribue à augmenter les revenus des villageois et par la même occasion à diminuer les défrichements et donc l'exploitation de nouveaux terrains en particulier dans les forêts où évoluent les lémuriens.

- le volet éducation : il vise à développer des activités de sensibilisation pour les communautés locales et à faciliter l'accès à l'éducation pour les enfants des villages impliqués dans le projet.

Ainsi Helpsimus soutient les 5 écoles primaires publiques localisées sur le site du programme Bamboo Lemur. En 5 ans, nous avons construit 6 salles de classe. Nous prenons également en charge la moitié du salaire de 14 enseignants et distribuons annuellement plus de 600 kits scolaires. Nous avons aussi financé la fabrication de 2 pirogues pour le transport des enfants et avons aménagé une bibliothèque. Enfin, nous avons ouvert en 2016 la première cantine scolaire de la région.

En 2013, Helpsimus a initié la « Fête du Simus » qui réunit chaque année l'ensemble des acteurs du programme Bamboo Lemur ainsi que les villages alentours autour de la préservation du grand hapalémur. Entre chaque discours officiel, les danseurs de « kilalaky » accompagnés par les chants de groupes locaux, font des démonstrations de cette danse traditionnelle très prisée à Madagascar. 


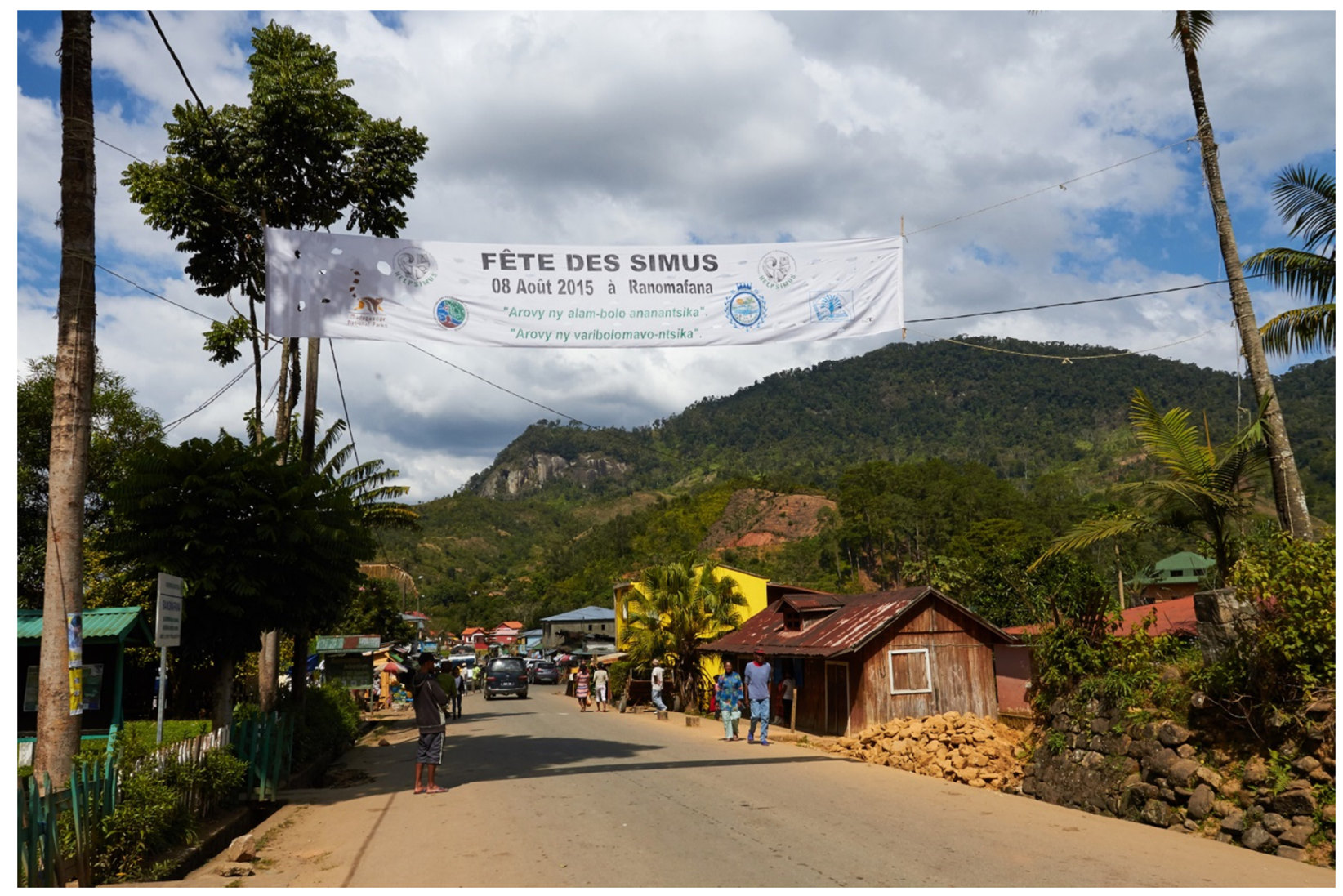

Photo 8. Fête du Simus (C) F. Perroux

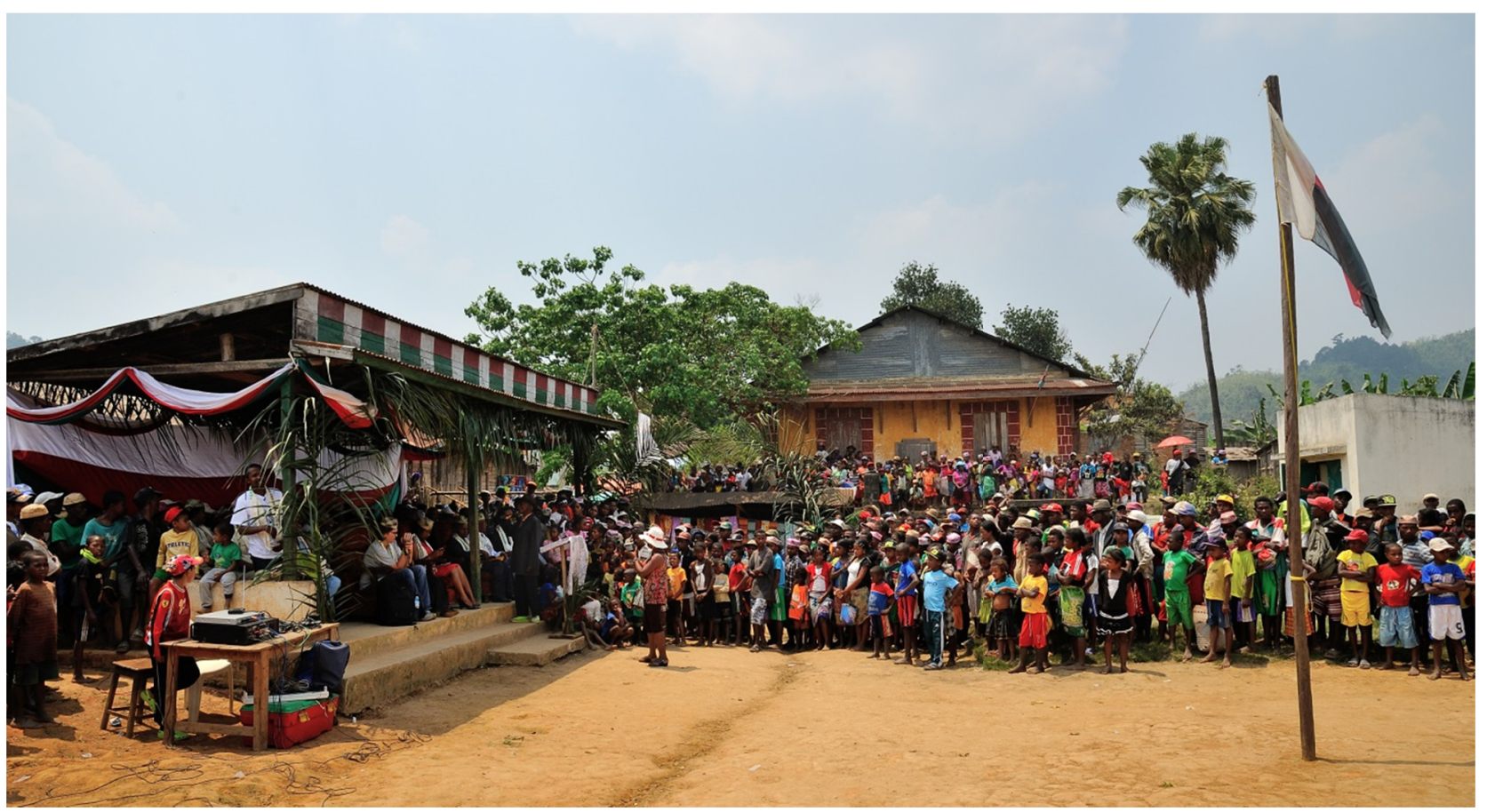

Photo 9. Fête du simus (c) F-G Grandin Helpsimus 


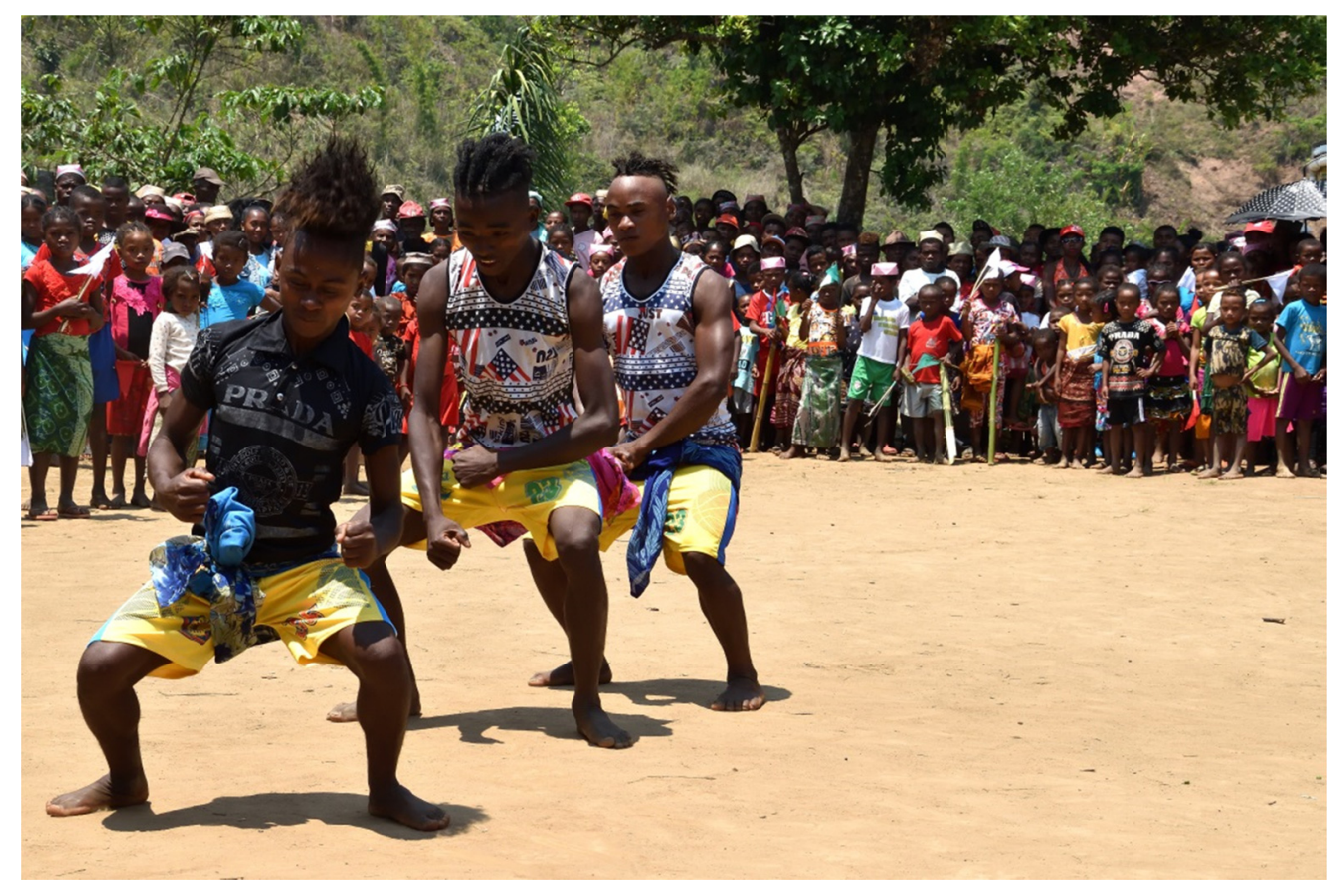

Photo 10. Les danseurs de kilalaky, Fête du simus @ D. Roullet

$\mathrm{Au}$ fil des ans cette fête est devenue un évènement incontournable dans la zone d'intervention d'Helpsimus et nous avons petit à petit cherché à développer des projets artistiques (théâtre, chant, danse...) accueillis avec enthousiasme par les écoles du projet.

A notre demande, les enfants de l'école de Sahofika, l'une des premières écoles soutenues par Helpsimus, ont préparé pour la $3^{\text {ème }}$ édition de la «Fête du Simus » un mini-spectacle original de chants et de danses sur le thème du grand hapalémur. On pouvait y voir le meneur des danses déguisé en lémurien. C'était la première fois que le grand hapalémur était mis en scène de cette façon. Et ce premier essai a ouvert la voie à d'autres créations artistiques qui sont devenues par la suite des outils de sensibilisation efficaces.

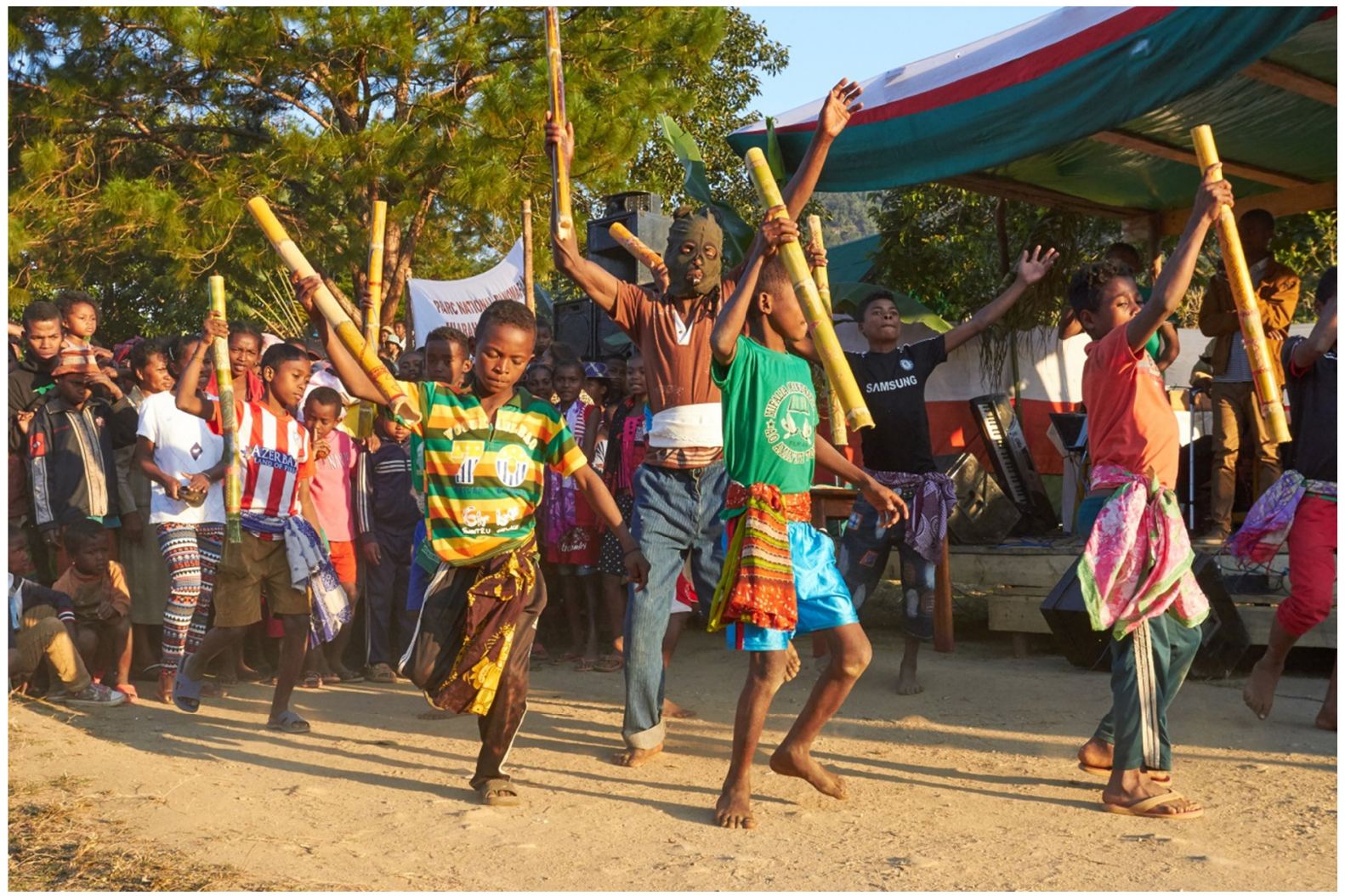

Photo 11. 1er spectacle de l'école de Sahofika, Fête du Simus @ F. Perroux 
Ensuite, chaque visite à l'école a été l'occasion pour les enfants de nous présenter de nouvelles créations : des chansons et des poèmes érigés en manifestes pour la préservation des forêts.

L'année suivante, nous avons demandé aux 5 écoles du programme de participer à la «Fête du Simus ». L'engouement était tel qu'il ne s'est d'ailleurs pas limité aux écoles. Des groupes artistiques se sont montés dans les villages dont les journées ont été rythmées par les répétitions.

Des masques de grand hapalémur ont été confectionnés et les «lamba » (pièce de tissu constituant un vêtement traditionnel malgache) ont été noués de telle sorte autour de la taille qu'on avait l'impression que les danseurs avaient une queue de lémurien.

Les enfants de l'école d'Ambodigoavy ont crée une mini-pièce de théâtre dont les situations comiques ont réjoui les spectateurs.

Cette 4ème édition de la «Fête du simus » fut un moment très particulier. Au-delà du plaisir évident pris par les participants, leurs créations ont illustré l'attachement des communautés villageoises aux grands hapalémurs et leur volonté réelle de les préserver. Une étape importante pour notre équipe qui a pu juger ce jour-là du chemin parcouru depuis 2008.

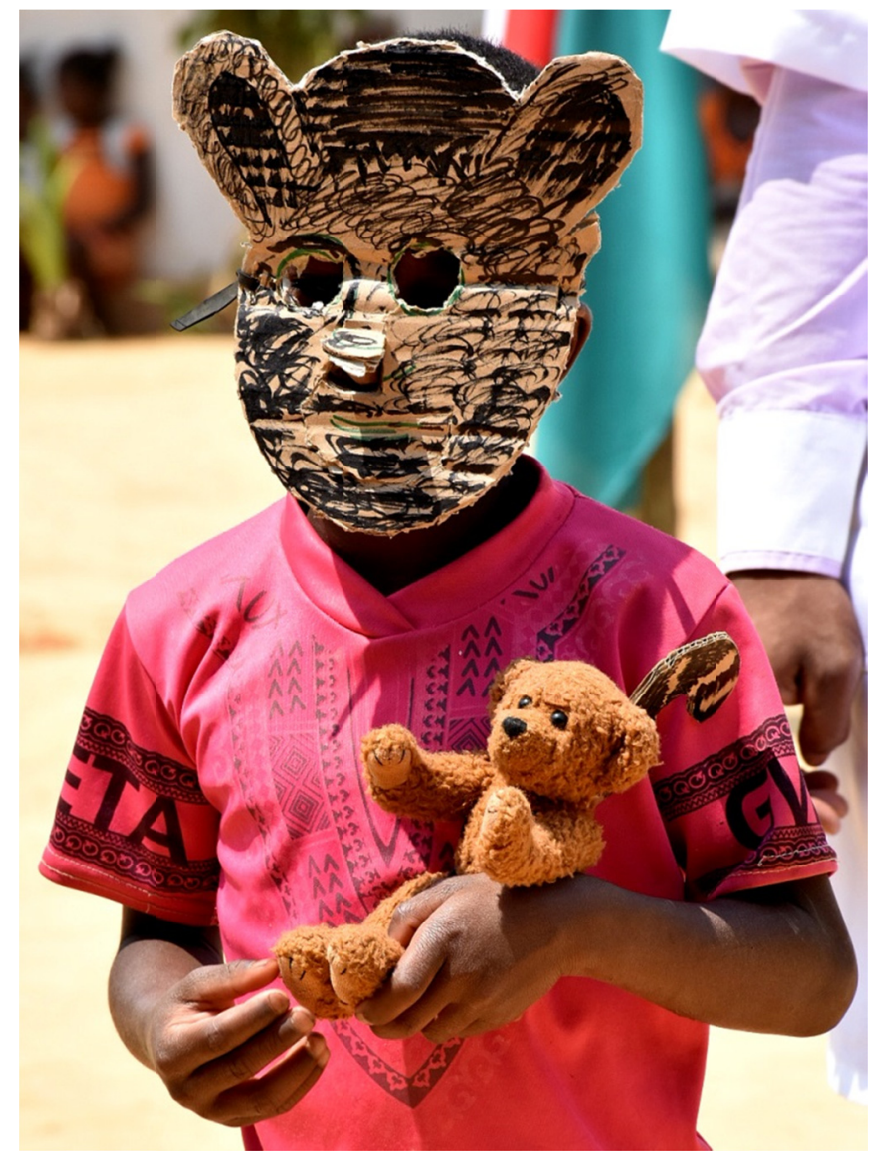

Photo 12. Spectacle des élèves de l'école d'Ambodigoavy à la fête du simus@ D. Roullet 


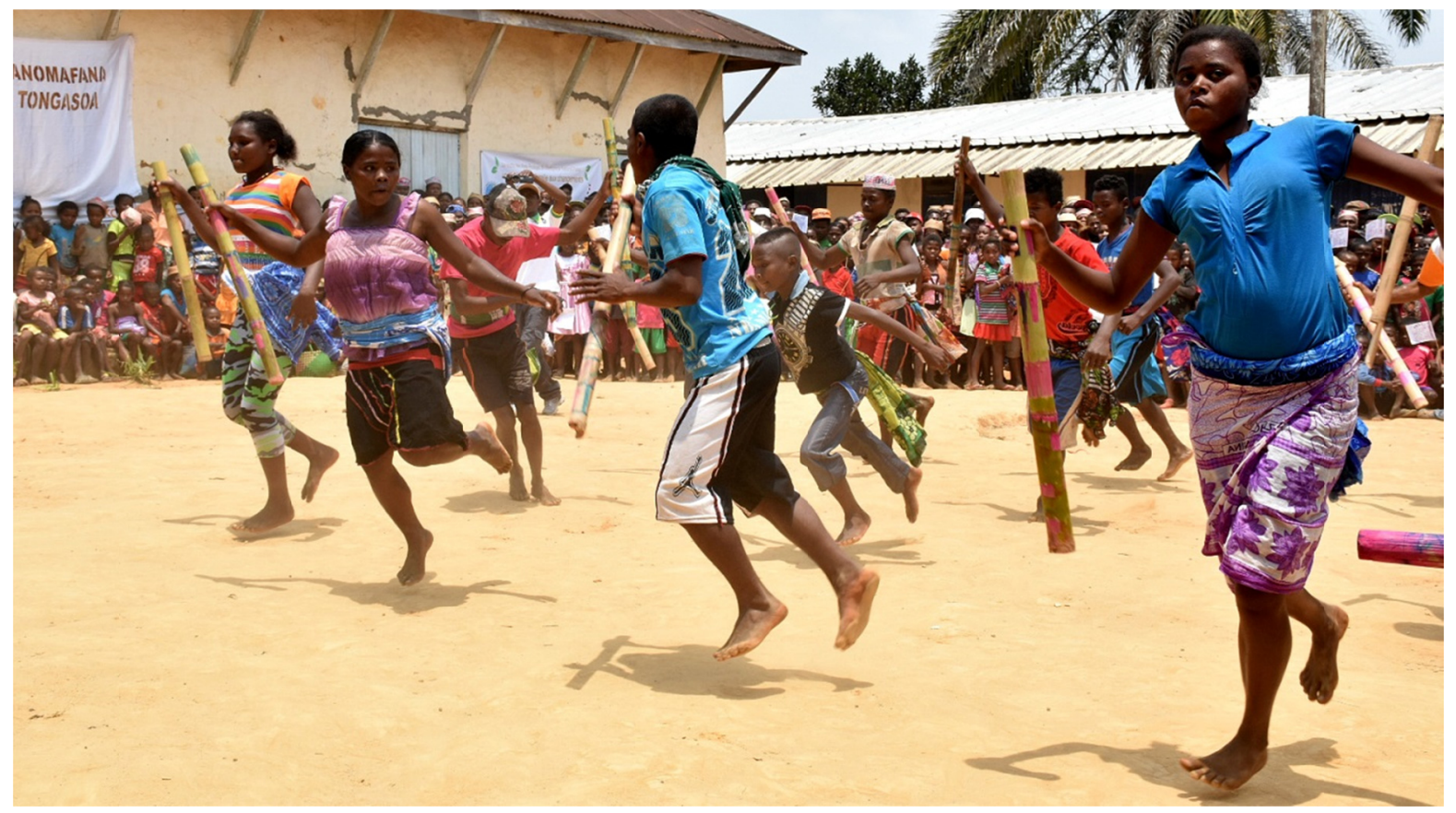

Photo 13. Le groupe de Volotora @ D. Roullet

Les évènements importants («Fête du Simus », inauguration de nouvelles structures, ritualisation de VOI etc.) que nous organisons régulièrement donnent désormais systématiquement l'opportunité à des groupes locaux de proposer des créations artistiques de plus en plus complexes. Ainsi les acrobaties des danseurs miment la vie des grands hapalémurs : une mère qui porte son jeune par exemple.

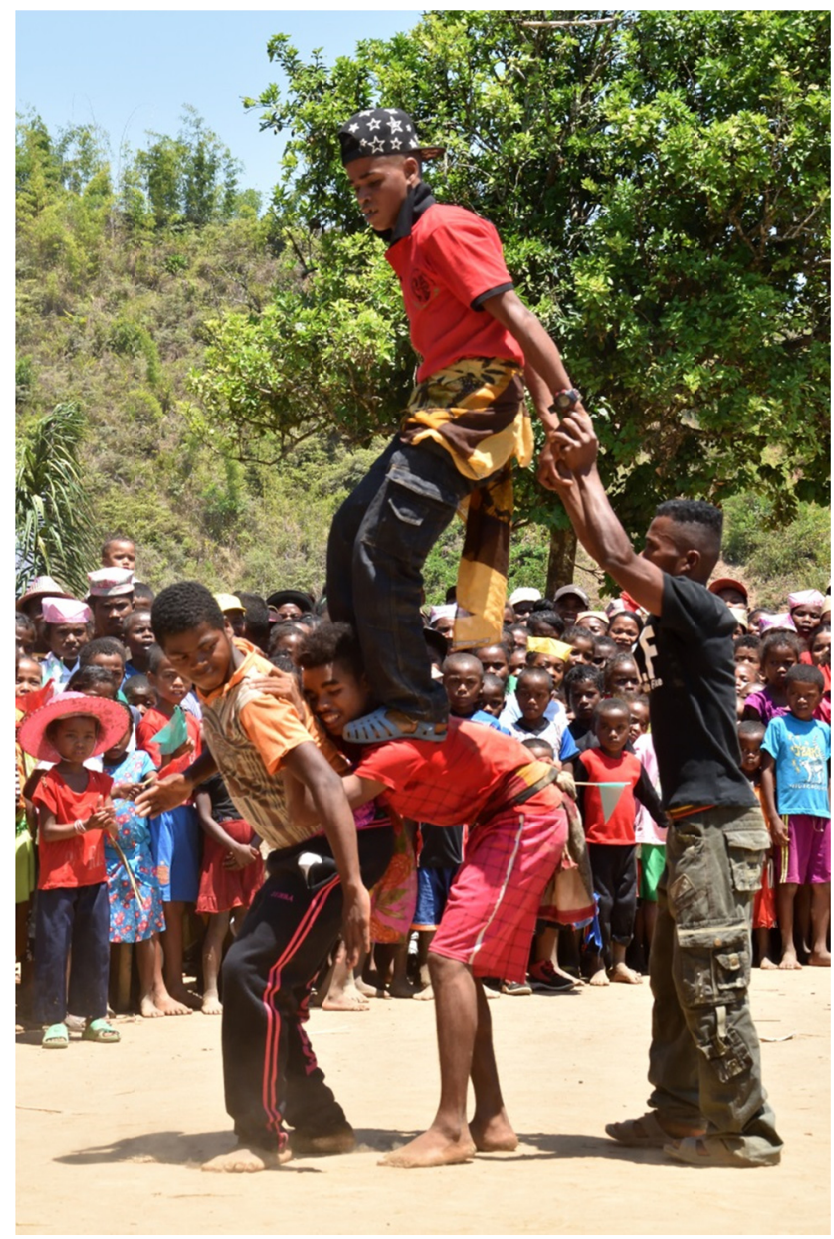

Photo 14. Acrobaties des danseurs Fête du simus@ D. Roullet 
En 2017, nous avons commandé à Menabe, un jeune peintre malgache originaire de Fianarantsoa, deux grandes fresques murales pour les 2 nouvelles salles de classe du village d'Ambodimanga dont la construction a été financée par Helpsimus. Celles-ci représentent des grands hapalémurs, leurs forêts de bambou et les villages à proximité desquels on peut les trouver. L'objectif était d'offrir aux enfants un lieu d'apprentissage agréable mais aussi de renforcer la présence des lémuriens dans leur quotidien.

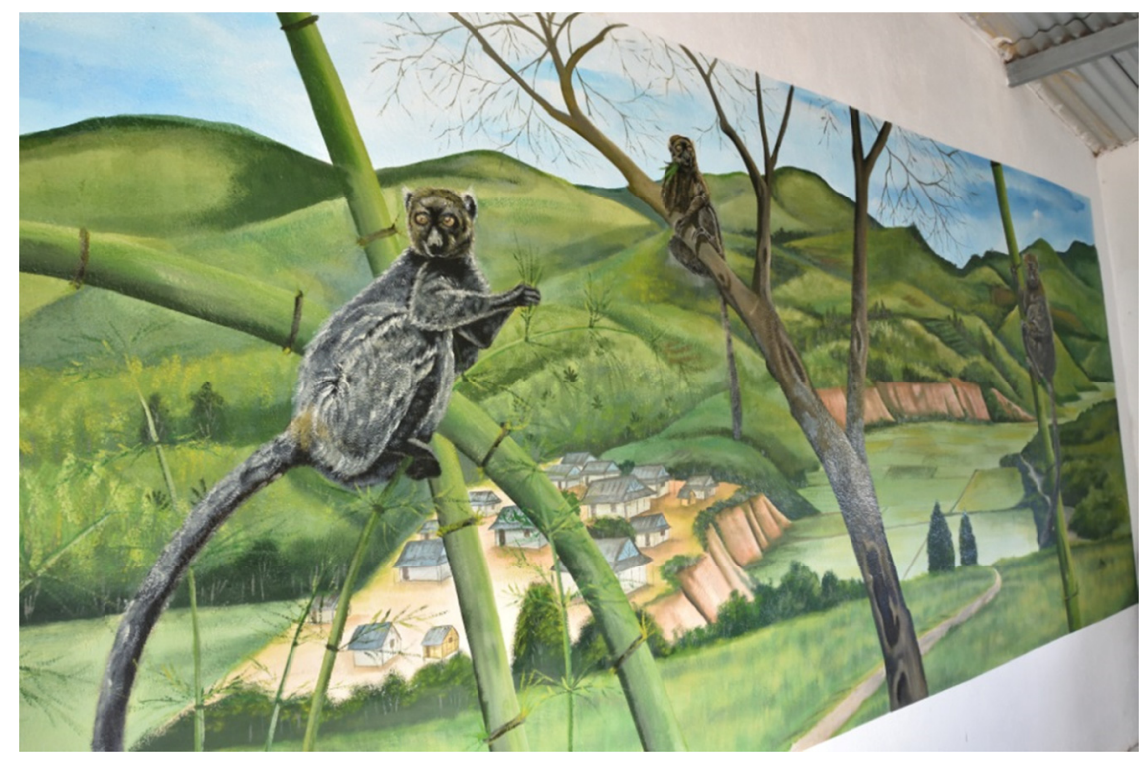

Photo 15. Fresque de Menaba à l'école d'Ambodimanga @ M. André

Encouragés par le succès de ces deux magnifiques œuvres, en particulier au moment de l'inauguration de l'école, nous avons réitéré l'expérience à l'école de Sahofika pour la $5^{\text {ème }}$ «Fête du Simus ». Cette fois nous avons demandé à Menabe de réaliser une performance artistique en terminant les 2 nouvelles fresques pendant la fête pour que les participants puissent l'observer en train de travailler. C'était la première fois qu'un artiste était mis en scène de cette façon et tous les participants ont pu apprécier son talent tout en voyant apparaître les lémuriens au fur et à mesure de l'évolution de son travail.

En 2015, les élèves de l'école de Sahofika ont démarré une correspondance avec ceux de l'école de Mervent localisée en Vendée. Pour raconter leur quotidien, les enfants ont souvent utilisé le dessin qui s'affranchit de la barrière du langage.

Les enfants français se sont alors rapidement rendus compte des difficultés auxquelles devaient faire face leurs camarades malgaches dans leur vie quotidienne. Pour les aider, ils leur ont fait parvenir via Helpsimus divers matériels obtenus à l'issue de plusieurs collectes qu'ils ont initiées. En 2017, ils ont organisé une fête malgache dans leur village dans le but de réunir suffisamment de fonds pour soutenir l'école de Sahofika et participer à l'achat de kits scolaires et au financement de la nouvelle cantine scolaire. Les élèves de CE2, CM1 et CM2 ont présenté une pièce de théâtre originale de leur création intitulée « Ecole Miracle » dans laquelle ils ont raconté avec beaucoup de poésie leur correspondance avec leurs camarades malgaches et ce qu'elle leur a apporté.

Grâce à des vidéos rapportées de Sahofika par l'une de leurs encadrantes, les enfants ont appris les danses et les chants traditionnels malgaches qu'ils ont présentés pendant cette fête. Pendant leur prestation, les films sur lesquels ils s'étaient entrainés montrant leurs camarades de Sahofika en train de danser, étaient projetés sur un écran à côté de la scène. 


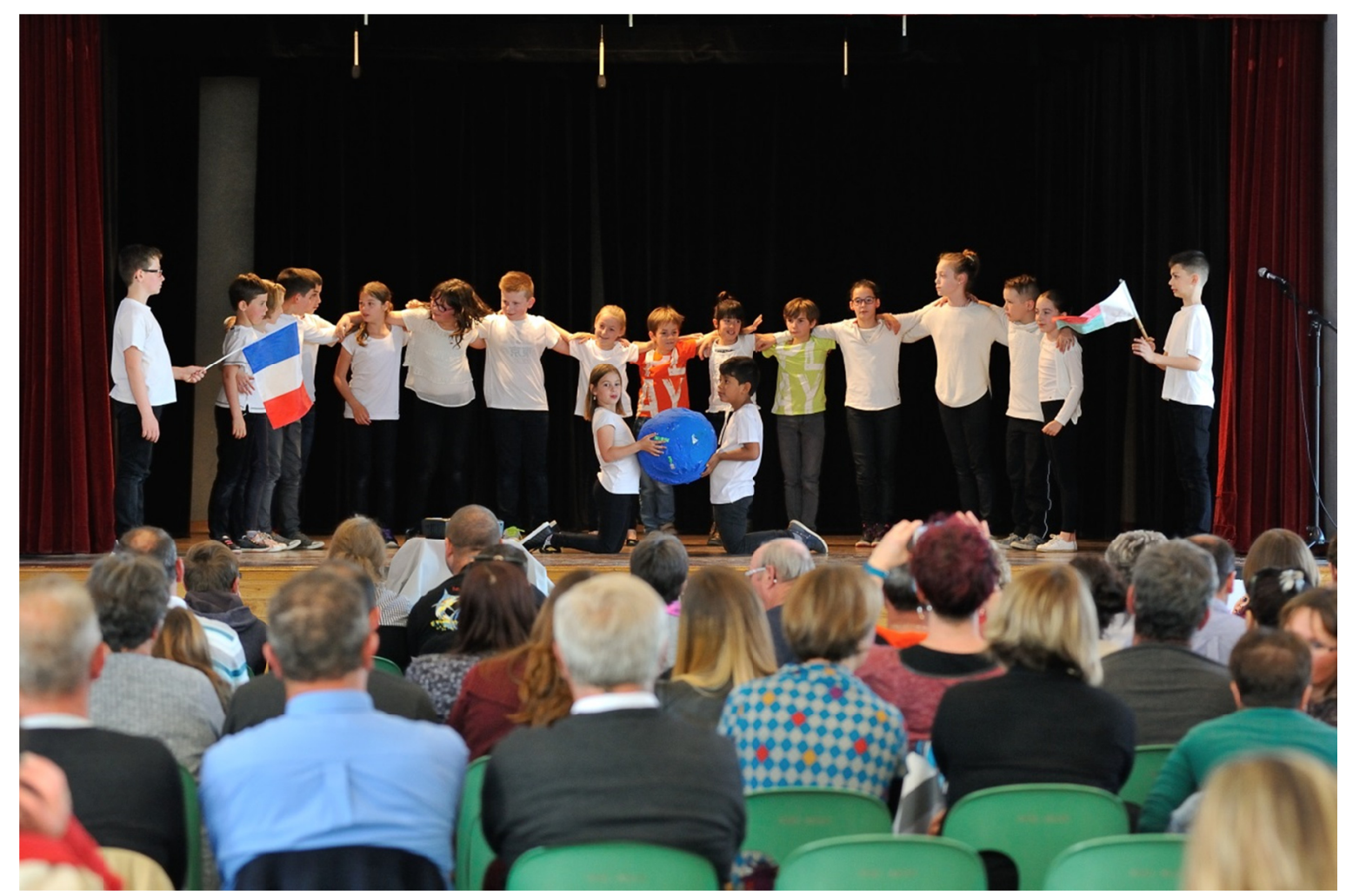

Photo 16. Pièce Ecole Miracle, Fête malgache Mervent ${ }^{\circledR} F-G$ Grandin

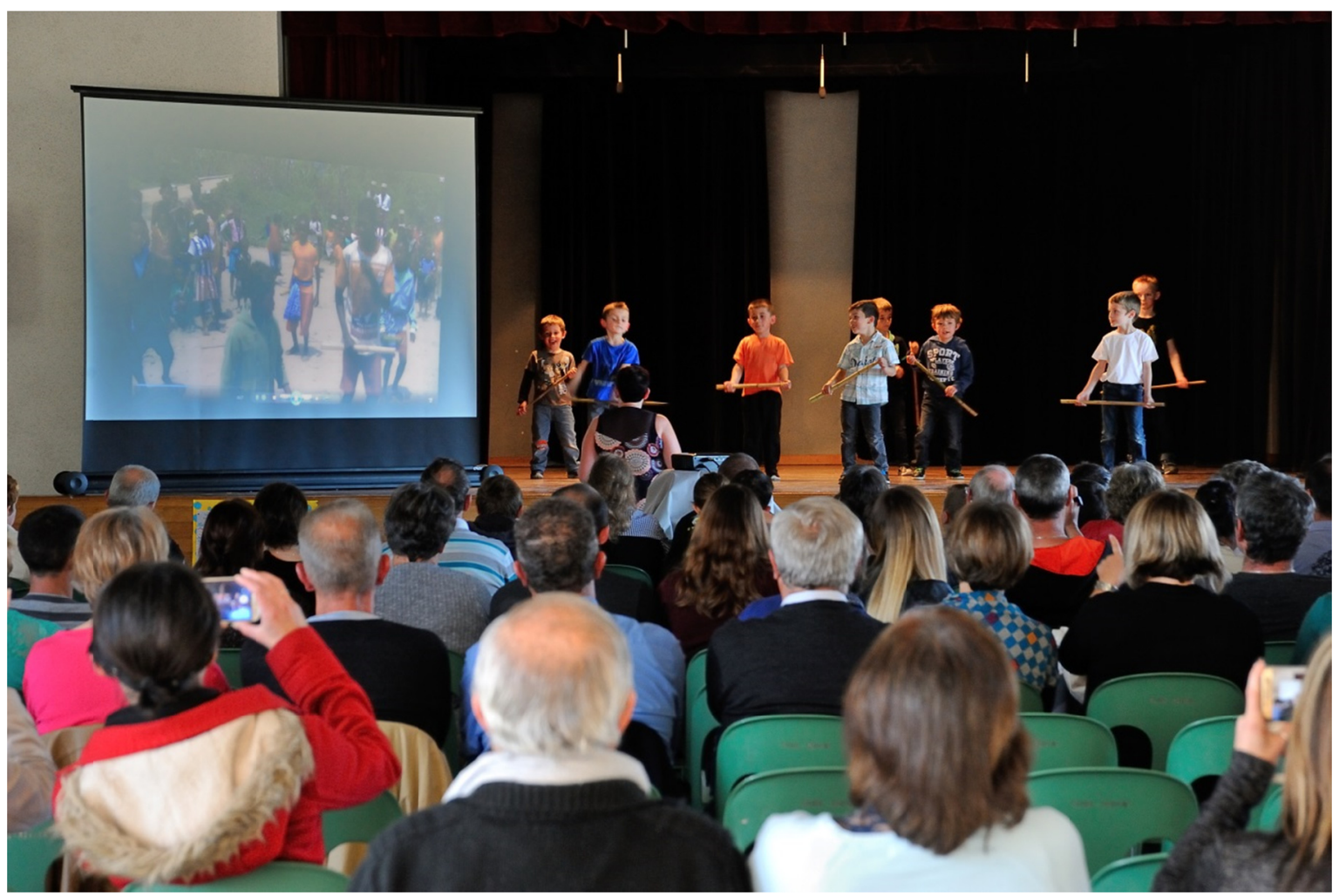

Photo 17. Fête malgache Mervent ${ }^{\circledR}$ F-G Grandin 


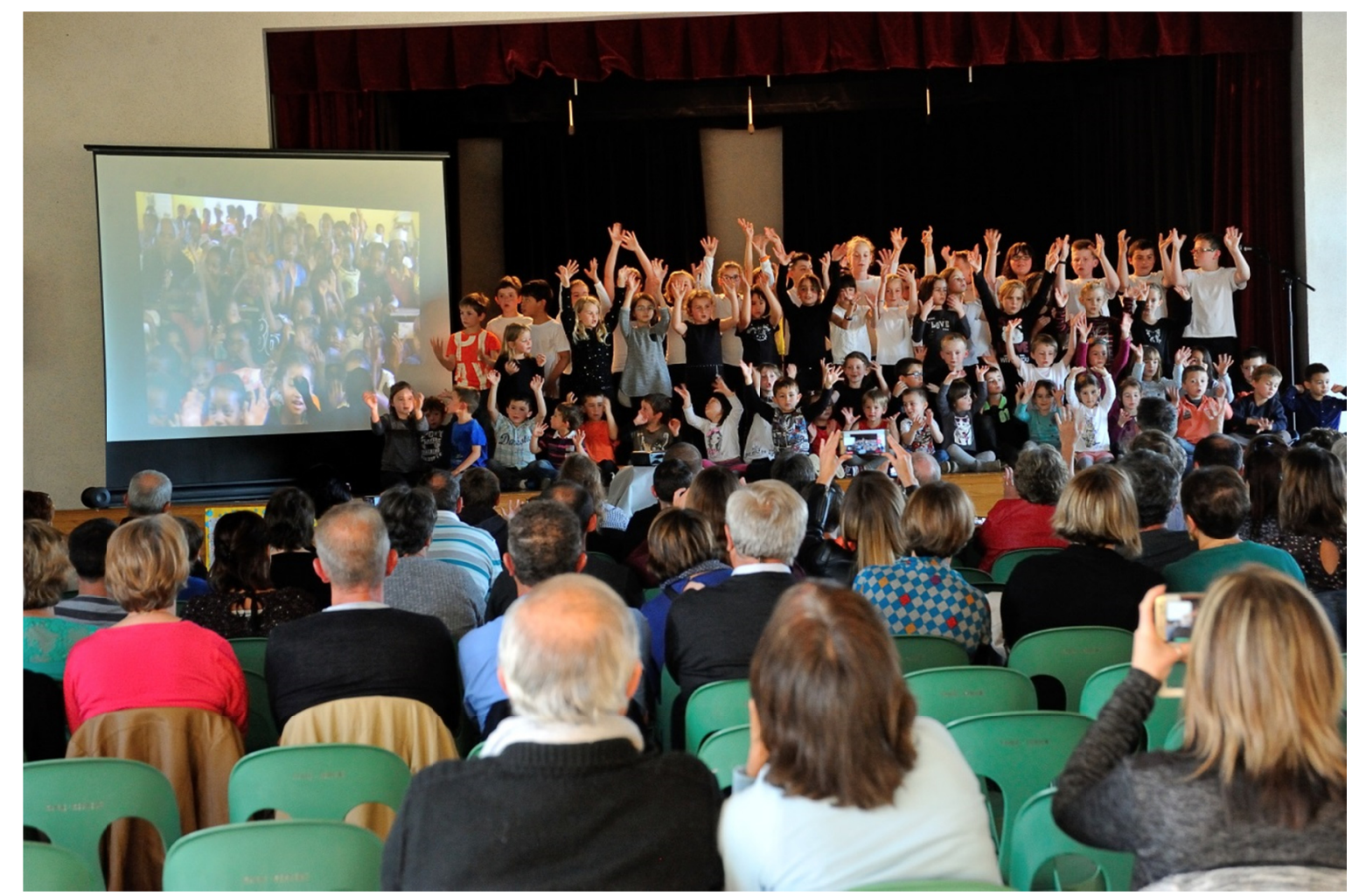

Photo 18. Fête malgache Mervent $®$ F-G Grandin

Nous avons également utilisé l'art dans nos actions de sensibilisation en France.

Ainsi en 2014, deux photographes animaliers ont monté pour le Festival International de photo animalière de Montier-en-Der, l'exposition photographique «Ce lémurien que l'on croyait perdu ». C'était la première exposition photographique entièrement consacrée au grand hapalémur et aux actions mises en œuvre par Helpsimus pour le sauvegarder.

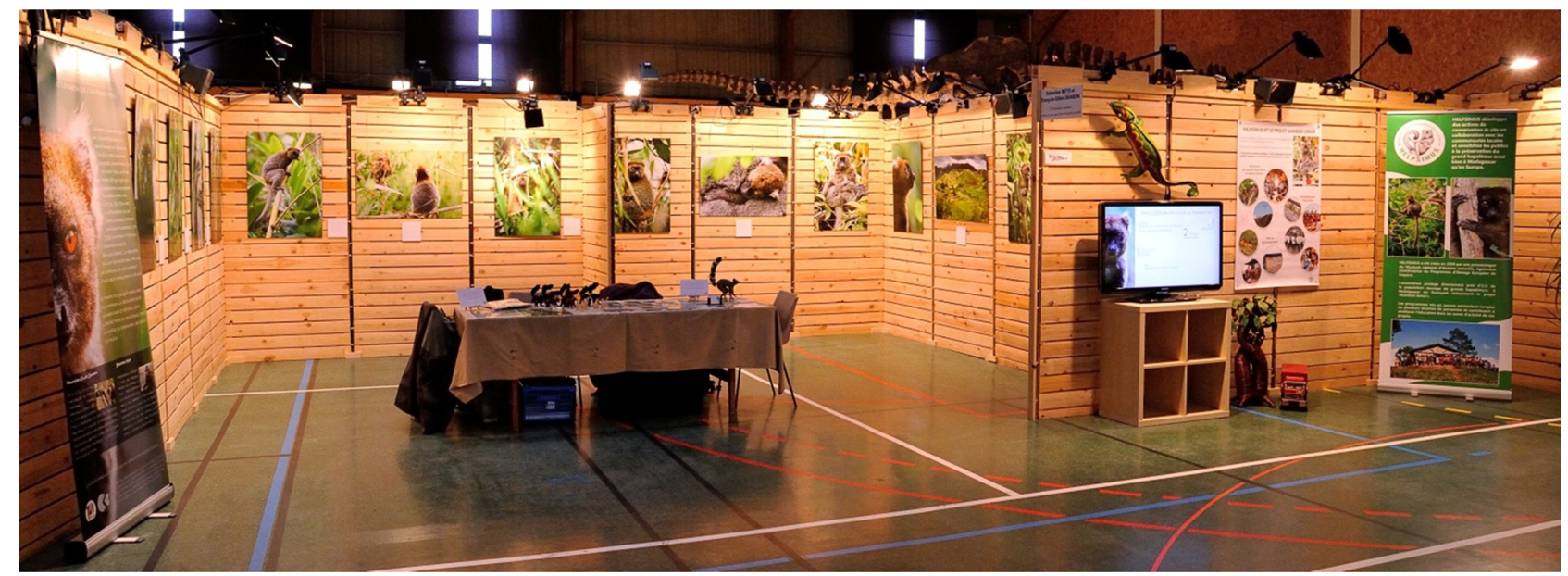

Photo 19. Exposition photo Montier en Der $®$ F-G Grandin Helpsimus

Trois années plus tard, elle a été présentée à la Galerie Louchard dans le cadre du festival des solidarités organisé par le $19^{\text {ème }}$ arrondissement de Paris. Trois artistes peintres l'ont enrichie de leurs œuvres. Parmi eux, Menabe, qui à l'occasion de sa première exposition en France, a réalisé plusieurs peintures de lémuriens sur des tronçons de bambou vernis. Cette exposition a notamment permis de toucher un public habitué aux galeries mais pas à la préservation de la biodiversité et a contrario d'ouvrir les portes de ce lieu à des personnes sensibilisées à la cause animale mais étrangères au monde de l'art dans un quartier parisien où les galeries sont rares. 


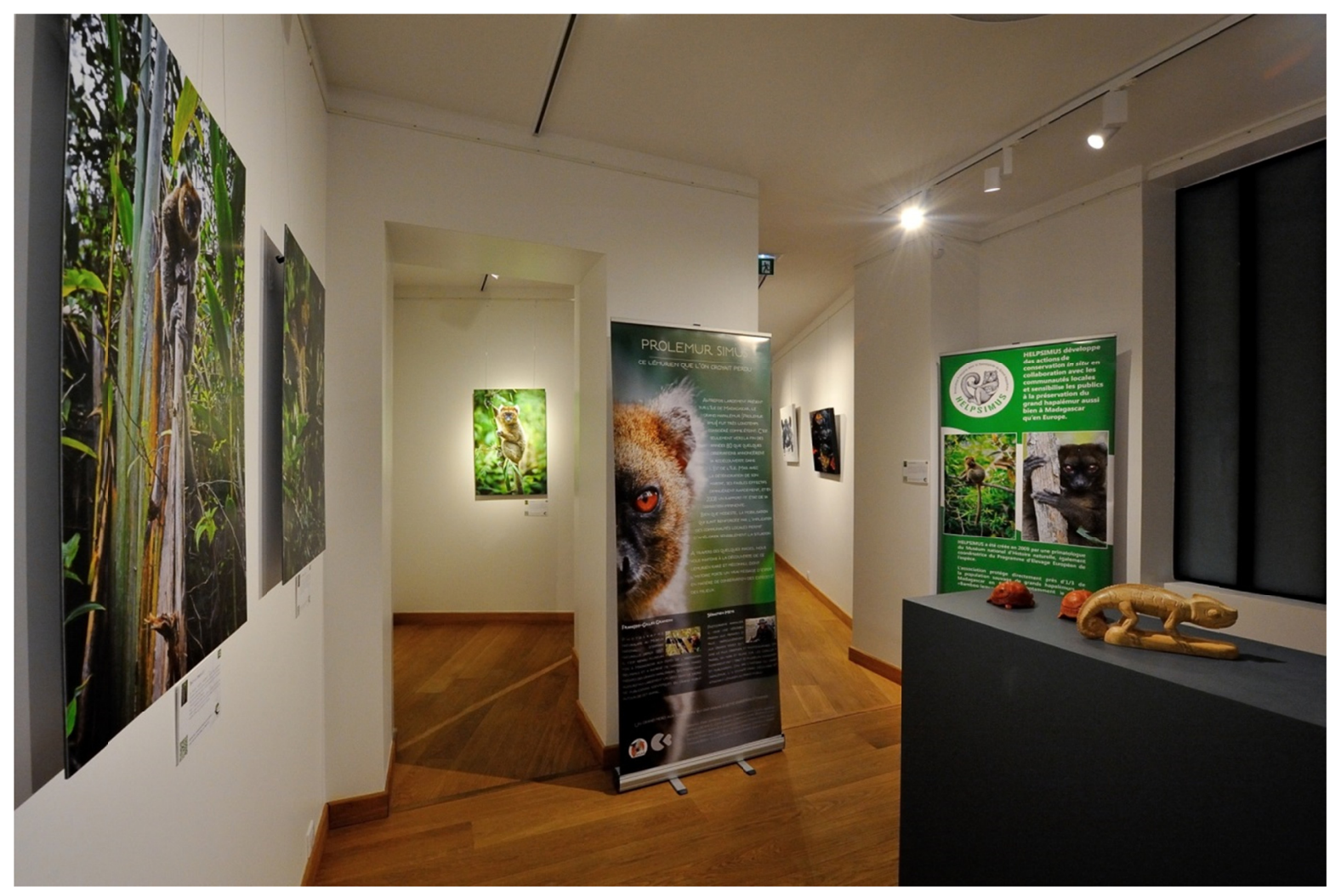

Photo 20. Exposition Galerie Louchard $®$ F-G Grandin

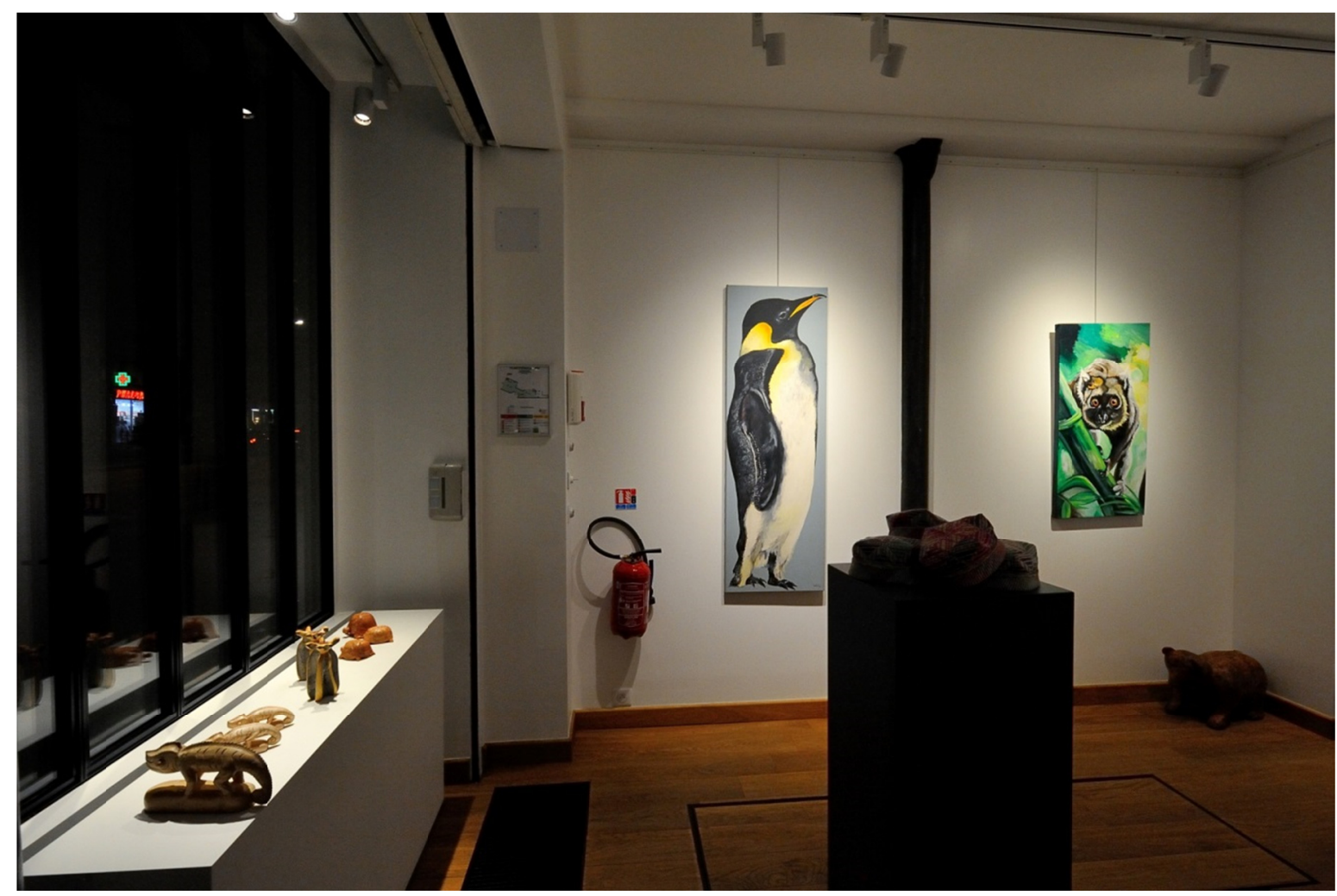

Photo 21. Exposition Galerie Louchard $®$ F-G Grandin

Enfin dans le cadre du projet d'écotourisme développé pour le programme Bamboo Lemur, Helpsimus a initié un projet artisanal incluant les femmes des villages avec lesquels nous collaborons.

Une créatrice de bijou française a imaginé pour ce projet un bracelet en graines de larme de job, une graminée qui pousse dans la région de notre programme. Elle formera prochainement plusieurs femmes 
à la fabrication de ce bijou. Elle créera également avec certaines d'entre elles un panier original en raphia. Ces produits seront vendus pour financer le programme.

Quand le programme Bamboo Lemur a démarré en 2008, nous n'avions pas idée de toutes les actions qui seraient mises en œuvre pour la protection de ce lémurien. Et nous ne pensions évidemment pas qu'il serait à l'origine de créations artistiques aussi nombreuses et variées qui ont non seulement participé à sa sauvegarde mais qui ont également permis de créer des liens entre des personnes d'origines différentes qui ne seraient probablement jamais rencontrées sans cet animal.

Les créations artistiques, du dessin d'un enfant à la peinture d'un artiste, ont fédéré de nombreuses personnes autour de la protection de ce lémurien, les impliquant personnellement et leur permettant de se l'approprier, souvent de façon ludique, partageant avec d'autres un but commun.

\section{Bibliographie}

Helpsimus (rapport non publié). 2015. 3rd International Technical Meeting on the conservation of the greater bamboo lemur(Prolemur Simus), 5-7 August 2015, Ranomafana, Madagascar.

Wright P. C., Johnson S. E., Irwin M. T., Jacobs R., Schlichting P., Lehman S., Louis Jr. E. E., Arrigo-Nelson S. J., Raharison J.-L., Rafalirarison R. R., Razafindratsita V., Ratsimbazafy J., Ratelolahy F. J., Dolch R. \& Tan C. 2008. The crisis of the Critically Endangered greater bamboo lemur (Prolemur simus). Primate Conservation 23: 5-17. 